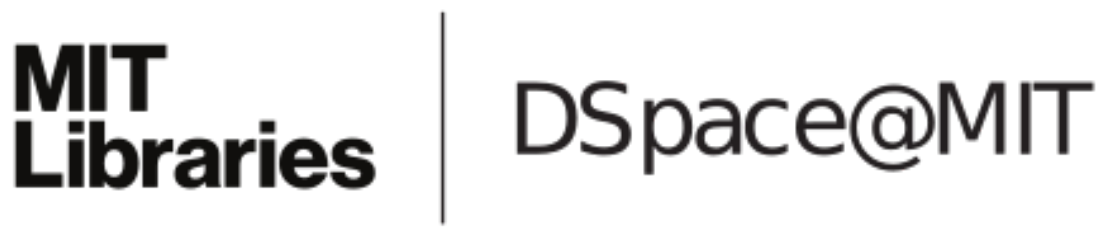

\author{
MIT Open Access Articles
}

Search for new particles decaying into dijets in proton-antiproton collisions at sqrt[s]=1.96 TeV

The MIT Faculty has made this article openly available. Please share how this access benefits you. Your story matters.

Citation: CDF Collaboration et al. "Search for new particles decaying into dijets in protonantiproton collisions at s=1.96 TeV." Physical Review D 79.11 (2009): 112002. (C) 2009 The American Physical Society

As Published: http://dx.doi.org/10.1103/PhysRevD.79.112002

Publisher: American Physical Society

Persistent URL: http://hdl.handle.net/1721.1/51851

Version: Final published version: final published article, as it appeared in a journal, conference proceedings, or other formally published context

Terms of Use: Article is made available in accordance with the publisher's policy and may be subject to US copyright law. Please refer to the publisher's site for terms of use. 


\section{Search for new particles decaying into dijets in proton-antiproton collisions at $\sqrt{s}=1.96 \mathrm{TeV}$}

T. Aaltonen, ${ }^{24}$ J. Adelman, ${ }^{14}$ T. Akimoto,${ }^{56}$ B. Álvarez González, ${ }^{12, \mathrm{r}}$ S. Amerio, ${ }^{44 \mathrm{~b}, 44 \mathrm{a}}$ D. Amidei, ${ }^{35}$ A. Anastassov,${ }^{39}$ A. Annovi, ${ }^{20}$ J. Antos, ${ }^{15}$ G. Apollinari,${ }^{18}$ A. Apresyan, ${ }^{49}$ T. Arisawa, ${ }^{58}$ A. Artikov, ${ }^{16}$ W. Ashmanskas, ${ }^{18}$ A. Attal, ${ }^{4}$ A. Aurisano,${ }^{54}$ F. Azfar, ${ }^{43}$ P. Azzurri, ${ }^{47 \mathrm{~d}, 47 \mathrm{a}}$ W. Badgett, ${ }^{18}$ A. Barbaro-Galtieri, ${ }^{29}$ V. E. Barnes, ${ }^{49}$ B. A. Barnett, ${ }^{26}$ V. Bartsch, ${ }^{31}$ G. Bauer, ${ }^{33}$ P.-H. Beauchemin, ${ }^{34}$ F. Bedeschi, ${ }^{47 a}$ D. Beecher,${ }^{31}$ S. Behari, ${ }^{26}$ G. Bellettini, ${ }^{47 b, 47 a}$ J. Bellinger,${ }^{60}$ D. Benjamin, ${ }^{17}$ A. Beretvas, ${ }^{18}$ J. Beringer, ${ }^{29}$ A. Bhatti, ${ }^{51}$ M. Binkley, ${ }^{18}$ D. Bisello, ${ }^{44 b, 44 a}$ I. Bizjak, ${ }^{31, w}$ R. E. Blair, ${ }^{2}$ C. Blocker, ${ }^{7}$ B. Blumenfeld, ${ }^{26}$ A. Bocci,,${ }^{17}$ A. Bodek, ${ }^{50}$ V. Boisvert, ${ }^{50}$ G. Bolla, ${ }^{49}$ D. Bortoletto, ${ }^{49}$ J. Boudreau ${ }^{48}$ A. Boveia, ${ }^{11}$ B. Brau, ${ }^{11, b}$ A. Bridgeman, ${ }^{25}$ L. Brigliadori, ${ }^{44 a}$ C. Bromberg, ${ }^{36}$ E. Brubaker, ${ }^{14}$ J. Budagov, ${ }^{16}$ H. S. Budd, ${ }^{50}$ S. Budd, ${ }^{25}$ S. Burke, ${ }^{18}$ K. Burkett, ${ }^{18}$ G. Busetto, ${ }^{44 b, 44 a}$ P. Bussey, ${ }^{22}$ A. Buzatu, ${ }^{34}$ K. L. Byrum, ${ }^{2}$ S. Cabrera,${ }^{17, t}$ C. Calancha, ${ }^{32}$ M. Campanelli, ${ }^{36}$ M. Campbell, ${ }^{35}$ F. Canelli, ${ }^{14,18}$ A. Canepa, ${ }^{46}$ B. Carls, ${ }^{25}$ D. Carlsmith,${ }^{60}$ R. Carosi, ${ }^{47 a}$ S. Carrillo, ${ }^{19, \mathrm{~m}}$ S. Carron, ${ }^{34}$ B. Casal, ${ }^{12}$ M. Casarsa, ${ }^{18}$ A. Castro, ${ }^{6 b, 6 a}$ P. Catastini,${ }^{47 c, 47 a}$ D. Cauz, ${ }^{55 b, 55 a}$ V. Cavaliere, ${ }^{47 c, 47 a}$ M. Cavalli-Sforza, ${ }^{4}$ A. Cerri, ${ }^{29}$ L. Cerrito,${ }^{31, n}$ S. H. Chang, ${ }^{28}$ Y. C. Chen, ${ }^{1}$ M. Chertok,${ }^{8}$ G. Chiarelli, ${ }^{47 a}$ G. Chlachidze, ${ }^{18}$ F. Chlebana, ${ }^{18}$ K. Cho, ${ }^{28}$ D. Chokheli, ${ }^{16}$ J. P. Chou, ${ }^{23}$ G. Choudalakis, ${ }^{33}$ S. H. Chuang, ${ }^{53}$ K. Chung, ${ }^{13}$ W. H. Chung, ${ }^{60}$ Y. S. Chung, ${ }^{50}$ T. Chwalek, ${ }^{27}$ C. I. Ciobanu, ${ }^{45}$ M. A. Ciocci, ${ }^{47 c, 47 a}$ A. Clark, ${ }^{21}$ D. Clark, ${ }^{7}$ G. Compostella, ${ }^{44 a}$ M. E. Convery, ${ }^{18}$ J. Conway, ${ }^{8}$ M. Cordelli, ${ }^{20}$ G. Cortiana,,${ }^{44 b, 44 a}$ C. A. Cox,${ }^{8}$ D. J. Cox,${ }^{8}$ F. Crescioli,,${ }^{47,47 a}$

C. Cuenca Almenar, ${ }^{8, \mathrm{t}} \mathrm{J}$. Cuevas, ${ }^{12, \mathrm{r}}$ R. Culbertson, ${ }^{18}$ J. C. Cully, ${ }^{35}$ D. Dagenhart, ${ }^{18}$ M. Datta, ${ }^{18}$ T. Davies, ${ }^{22}$ P. de Barbaro, ${ }^{50}$ S. De Cecco, ${ }^{52 a}$ A. Deisher, ${ }^{29}$ G. De Lorenzo, ${ }^{4}$ M. Dell'Orso, ${ }^{47 b, 47 a}$ C. Deluca, ${ }^{4}$ L. Demortier,${ }^{51}$ J. Deng, ${ }^{17}$ M. Deninno, ${ }^{6 a}$ P. F. Derwent, ${ }^{18}$ G. P. di Giovanni, ${ }^{45}$ C. Dionisi, ${ }^{52 b, 52 a}$ B. Di Ruzza, ${ }^{55 b, 55 a}$ J. R. Dittmann, ${ }^{5}$ M. D’Onofrio, ${ }^{4}$ S. Donati, ${ }^{47 b, 47 a}$ P. Dong, ${ }^{9}$ J. Donini, ${ }^{44 a}$ T. Dorigo, ${ }^{44 a}$ S. Dube, ${ }^{53}$ J. Efron ${ }^{40}$ A. Elagin,${ }^{54}$ R. Erbacher, ${ }^{8}$ D. Errede, ${ }^{25}$ S. Errede,${ }^{25}$ R. Eusebi, ${ }^{18}$ H. C. Fang, ${ }^{29}$ S. Farrington, ${ }^{43}$ W. T. Fedorko, ${ }^{14}$ R. G. Feild, ${ }^{61}$ M. Feindt, ${ }^{27}$ J. P. Fernandez,${ }^{32}$ C. Ferrazza ${ }^{47 \mathrm{~d}, 47 \mathrm{a}}$ R. Field,${ }^{19}$ G. Flanagan,${ }^{49}$ R. Forrest,${ }^{8}$ M. J. Frank, ${ }^{5}$ M. Franklin, ${ }^{23}$ J. C. Freeman, ${ }^{18}$ I. Furic, ${ }^{19}$ M. Gallinaro, ${ }^{52 a}$ J. Galyardt, ${ }^{13}$ F. Garberson, ${ }^{11}$ J. E. Garcia, ${ }^{21}$ A. F. Garfinkel,${ }^{49}$ K. Genser,${ }^{18}$ H. Gerberich, ${ }^{25}$ D. Gerdes ${ }^{35}$ A. Gessler, ${ }^{27}$ S. Giagu, ${ }^{52 b, 52 a}$ V. Giakoumopoulou, ${ }^{3}$ P. Giannetti, ${ }^{47 a}$ K. Gibson, ${ }^{48}$ J. L. Gimmell, ${ }^{50}$ C. M. Ginsburg, ${ }^{18}$ N. Giokaris, ${ }^{3}$ M. Giordani, ${ }^{55 b, 55 a}$ P. Giromini, ${ }^{20}$ M. Giunta, ${ }^{47 b, 47 a}$ G. Giurgiu, ${ }^{26}$ V. Glagolev,${ }^{16}$ D. Glenzinski, ${ }^{18}$ M. Gold, ${ }^{38}$ N. Goldschmidt ${ }^{19}$ A. Golossanov, ${ }^{18}$ G. Gomez, ${ }^{12}$ G. Gomez-Ceballos, ${ }^{33}$ M. Goncharov, ${ }^{33}$ O. González, ${ }^{32}$ I. Gorelov, ${ }^{38}$ A. T. Goshaw, ${ }^{17}$ K. Goulianos, ${ }^{51}$ A. Gresele, ${ }^{44 b, 44 a}$ S. Grinstein, ${ }^{23}$ C. Grosso-Pilcher, ${ }^{14}$ R. C. Group,${ }^{18}$ U. Grundler, ${ }^{25}$ J. Guimaraes da Costa, ${ }^{23}$ Z. Gunay-Unalan, ${ }^{36}$ C. Haber, ${ }^{29}$ K. Hahn, ${ }^{33}$ S. R. Hahn, ${ }^{18}$ E. Halkiadakis, ${ }^{53}$ B.-Y. Han, ${ }^{50}$ J. Y. Han,${ }^{50}$ F. Happacher ${ }^{20}$ K. Hara,${ }^{56}$ D. Hare,${ }^{53}$ M. Hare, ${ }^{57}$ S. Harper ${ }^{43}$ R. F. Harr, ${ }^{59}$ R. M. Harris,${ }^{18}$ M. Hartz ${ }^{48}$ K. Hatakeyama, ${ }^{51}$ C. Hays, ${ }^{43}$ M. Heck,${ }^{27}$ A. Heijboer, ${ }^{46}$ J. Heinrich, ${ }^{46}$ C. Henderson, ${ }^{33}$ M. Herndon, ${ }^{60}$ J. Heuser,${ }^{27}$ S. Hewamanage, ${ }^{5}$ D. Hidas, ${ }^{17}$ C. S. Hill, ${ }^{11, \mathrm{~d}}$ D. Hirschbuehl,${ }^{27}$ A. Hocker, ${ }^{18}$ S. Hou, ${ }^{1}$ M. Houlden,${ }^{30}$ S.-C. Hsu, ${ }^{29}$ B. T. Huffman, ${ }^{43}$ R. E. Hughes, ${ }^{40}$ U. Husemann, ${ }^{61}$ M. Hussein, ${ }^{36}$ J. Huston, ${ }^{36}$ J. Incandela, ${ }^{11}$ G. Introzzi, ${ }^{47 a}$ M. Iori, ${ }^{52 b, 52 a}$ A. Ivanov, ${ }^{8}$ E. James, ${ }^{18}$ D. Jang, ${ }^{13}$ B. Jayatilaka, ${ }^{17}$ E. J. Jeon, ${ }^{28}$ M. K. Jha, ${ }^{6 a}$ S. Jindariani, ${ }^{18}$ W. Johnson, ${ }^{8}$ M. Jones ${ }^{49}$ K. K. Joo, ${ }^{28}$ S. Y. Jun, ${ }^{13}$ J. E. Jung, ${ }^{28}$ T. R. Junk,,${ }^{18}$ T. Kamon, ${ }^{54}$ D. Kar, ${ }^{19}$ P. E. Karchin,${ }^{59}$ Y. Kato, ${ }^{42}$ R. Kephart, ${ }^{18}$ J. Keung, ${ }^{46}$ V. Khotilovich, ${ }^{54}$ B. Kilminster, ${ }^{18}$ D. H. Kim, ${ }^{28}$ H. S. Kim, ${ }^{28}$ H. W. Kim, ${ }^{28}$ J. E. Kim, ${ }^{28}$ M. J. Kim, ${ }^{20}$ S. B. Kim, ${ }^{28}$ S. H. Kim, ${ }^{56}$ Y. K. Kim, ${ }^{14}$ N. Kimura,${ }^{56}$ L. Kirsch, ${ }^{7}$ S. Klimenko, ${ }^{19}$ B. Knuteson, ${ }^{33}$ B. R. Ko, ${ }^{17}$ K. Kondo, ${ }^{58}$ D. J. Kong, ${ }^{28}$ J. Konigsberg, ${ }^{19}$ A. Korytov, ${ }^{19}$ A. V. Kotwal, ${ }^{17}$ M. Kreps,${ }^{27}$ J. Kroll, ${ }^{46}$ D. Krop,${ }^{14}$ N. Krumnack,,${ }^{5}$ M. Kruse, ${ }^{17}$ V. Krutelyov, ${ }^{11}$ T. Kubo, ${ }^{56}$ T. Kuhr, ${ }^{27}$ N.P. Kulkarni ${ }^{59}$ M. Kurata, ${ }^{56}$ S. Kwang, ${ }^{14}$ A. T. Laasanen, ${ }^{49}$ S. Lami, ${ }^{47 a}$ S. Lammel, ${ }^{18}$ M. Lancaster, ${ }^{31}$ R. L. Lander, ${ }^{8}$ K. Lannon, ${ }^{40, \mathrm{q}}$ A. Lath, ${ }^{53}$ G. Latino, ${ }^{47 c, 47 a}$ I. Lazzizzera, ${ }^{44 b, 44 a}$ T. LeCompte, ${ }^{2}$ E. Lee ${ }^{54}$ H. S. Lee, ${ }^{14}$ S. W. Lee, ${ }^{54, s}$ S. Leone, ${ }^{47 a}$ J. D. Lewis, ${ }^{18}$ C.-S. Lin,${ }^{29}$ J. Linacre, ${ }^{43}$ M. Lindgren, ${ }^{18}$ E. Lipeles, ${ }^{46}$ A. Lister, ${ }^{8}$ D. O. Litvintsev, ${ }^{18}$ C. Liu, ${ }^{48}$ T. Liu, ${ }^{18}$ N. S. Lockyer, ${ }^{46}$ A. Loginov,${ }^{61}$ M. Loreti, ${ }^{44 b, 44 a}$ L. Lovas, ${ }^{15}$ D. Lucchesi ${ }^{44 b, 44 a}$ C. Luci, ${ }^{52 b, 52 a}$ J. Lueck,${ }^{27}$ P. Lujan, ${ }^{29}$ P. Lukens, ${ }^{18}$ G. Lungu, ${ }^{51}$ L. Lyons, ${ }^{43}$ J. Lys, ${ }^{29}$ R. Lysak,${ }^{15}$ D. MacQueen, ${ }^{34}$ R. Madrak,${ }^{18}$ K. Maeshima, ${ }^{18}$ K. Makhoul, ${ }^{33}$ T. Maki ${ }^{24}$ P. Maksimovic,${ }^{26}$ S. Malde, ${ }^{43}$ S. Malik, ${ }^{31}$

G. Manca, ${ }^{30, f}$ A. Manousakis-Katsikakis, ${ }^{3}$ F. Margaroli, ${ }^{49}$ C. Marino, ${ }^{27}$ C. P. Marino, ${ }^{25}$ A. Martin, ${ }^{61}$ V. Martin, ${ }^{22,1}$ M. Martínez, ${ }^{4}$ R. Martínez-Ballarín, ${ }^{32}$ T. Maruyama, ${ }^{56}$ P. Mastrandrea, ${ }^{52 a}$ T. Masubuchi, ${ }^{56}$ M. Mathis,${ }^{26}$ M. E. Mattson, ${ }^{59}$ P. Mazzanti, ${ }^{6 a}$ K. S. McFarland ${ }^{50}$ P. McIntyre, ${ }^{54}$ R. McNulty,${ }^{30, k}$ A. Mehta ${ }^{30}$ P. Mehtala, ${ }^{24}$ A. Menzione, ${ }^{47 a}$ P. Merkel, ${ }^{49}$ C. Mesropian, ${ }^{51}$ T. Miao, ${ }^{18}$ N. Miladinovic, ${ }^{7}$ R. Miller, ${ }^{36}$ C. Mills,${ }^{23}$ M. Milnik,${ }^{27}$ A. Mitra, ${ }^{1}$ G. Mitselmakher,${ }^{19}$

H. Miyake, ${ }^{56}$ N. Moggi, ${ }^{6 a}$ C. S. Moon, ${ }^{28}$ R. Moore, ${ }^{18}$ M. J. Morello, ${ }^{47 b, 47 a}$ J. Morlock, ${ }^{27}$ P. Movilla Fernandez,${ }^{18}$ J. Mülmenstädt, ${ }^{29}$ A. Mukherjee, ${ }^{18}$ Th. Muller,${ }^{27}$ R. Mumford,${ }^{26}$ P. Murat,,${ }^{18}$ M. Mussini, ${ }^{6 b, 6 a}$ J. Nachtman, ${ }^{18}$ Y. Nagai, ${ }^{56}$ A. Nagano, ${ }^{56}$ J. Naganoma, ${ }^{56}$ K. Nakamura, ${ }^{56}$ I. Nakano, ${ }^{41}$ A. Napier, ${ }^{57}$ V. Necula, ${ }^{17}$ J. Nett, ${ }^{60}$ C. Neu, ${ }^{46, u}$ M. S. Neubauer, ${ }^{25}$ S. Neubauer, ${ }^{27}$ J. Nielsen, ${ }^{29, h}$ L. Nodulman, ${ }^{2}$ M. Norman, ${ }^{10}$ O. Norniella, ${ }^{25}$ E. Nurse,${ }^{31}$ L. Oakes, ${ }^{43}$ 
S. H. Oh, ${ }^{17}$ Y.D. Oh, ${ }^{28}$ I. Oksuzian, ${ }^{19}$ T. Okusawa, ${ }^{42}$ R. Orava, ${ }^{24}$ K. Osterberg, ${ }^{24}$ S. Pagan Griso, ${ }^{44 b, 44 a}$ E. Palencia, ${ }^{18}$ V. Papadimitriou, ${ }^{18}$ A. Papaikonomou, ${ }^{27}$ A. A. Paramonov, ${ }^{14}$ B. Parks, ${ }^{40}$ S. Pashapour, ${ }^{34}$ J. Patrick, ${ }^{18}$ G. Pauletta, ${ }^{55 b, 55 a}$ M. Paulini, ${ }^{13}$ C. Paus, ${ }^{33}$ T. Peiffer,${ }^{27}$ D. E. Pellett, ${ }^{8}$ A. Penzo ${ }^{55 a}$ T. J. Phillips,${ }^{17}$ G. Piacentino, ${ }^{47 a}$ E. Pianori,${ }^{46}$ L. Pinera, ${ }^{19}$ K. Pitts, ${ }^{25}$ C. Plager, ${ }^{9}$ L. Pondrom, ${ }^{60}$ O. Poukhov, ${ }^{16, a}$ N. Pounder, ${ }^{43}$ F. Prakoshyn, ${ }^{16}$ A. Pronko, ${ }^{18}$ J. Proudfoot, ${ }^{2}$ F. Ptohos, ${ }^{18, j}$ E. Pueschel,${ }^{13}$ G. Punzi,${ }^{47 b, 47 a}$ J. Pursley,${ }^{60}$ J. Rademacker, ${ }^{43, d}$ A. Rahaman, ${ }^{48}$ V. Ramakrishnan, ${ }^{60}$ N. Ranjan, ${ }^{49}$ I. Redondo, ${ }^{32}$ P. Renton, ${ }^{43}$ M. Renz,${ }^{27}$ M. Rescigno, ${ }^{52 a}$ S. Richter, ${ }^{27}$ F. Rimondi, ${ }^{6 b, 6 a}$ L. Ristori,${ }^{47 a}$ A. Robson, ${ }^{22}$ T. Rodrigo, ${ }^{12}$ T. Rodriguez, ${ }^{46}$ E. Rogers,${ }^{25}$ S. Rolli, ${ }^{57}$ R. Roser, ${ }^{18}$ M. Rossi, ${ }^{55 a}$ R. Rossin, ${ }^{11}$ P. Roy, ${ }^{34}$ A. Ruiz, ${ }^{12}$ J. Russ, ${ }^{13}$ V. Rusu, ${ }^{18}$ H. Saarikko, ${ }^{24}$ A. Safonov, ${ }^{54}$ W. K. Sakumoto, ${ }^{50}$ O. Saltó, ${ }^{4}$ L. Santi, ${ }^{55 b, 55 a}$ S. Sarkar, ${ }^{52 b, 52 a}$ L. Sartori, ${ }^{47 a}$ K. Sato, ${ }^{18}$ A. Savoy-Navarro, ${ }^{45}$ P. Schlabach,${ }^{18}$ A. Schmidt, ${ }^{27}$ E. E. Schmidt, ${ }^{18}$ M. A. Schmidt,${ }^{14}$ M. P. Schmidt, ${ }^{61, a}$ M. Schmitt, ${ }^{39}$ T. Schwarz, ${ }^{8}$ L. Scodellaro, ${ }^{12}$ A. Scribano, ${ }^{47 c, 47 a}$ F. Scuri,${ }^{47 a}$ A. Sedov, ${ }^{49}$ S. Seidel, ${ }^{38}$ Y. Seiya, ${ }^{42}$ A. Semenov, ${ }^{16}$ L. Sexton-Kennedy, ${ }^{18}$ F. Sforza, ${ }^{47 a}$ A. Sfyrla, ${ }^{25}$ S. Z. Shalhout, ${ }^{59}$ T. Shears, ${ }^{30}$ P. F. Shepard, ${ }^{48}$ M. Shimojima, ${ }^{56, p}$ S. Shiraishi, ${ }^{14}$ M. Shochet, ${ }^{14}$ Y. Shon, ${ }^{60}$ I. Shreyber,${ }^{37}$ A. Sidoti, ${ }^{47 a}$ P. Sinervo, ${ }^{34}$ A. Sisakyan, ${ }^{16}$ A. J. Slaughter, ${ }^{18}$ J. Slaunwhite, ${ }^{40}$ K. Sliwa, ${ }^{57}$ J. R. Smith, ${ }^{8}$ F. D. Snider, ${ }^{18}$ R. Snihur,${ }^{34}$ A. Soha, ${ }^{8}$ S. Somalwar, ${ }^{53}$ V. Sorin, ${ }^{36}$ J. Spalding, ${ }^{18}$ T. Spreitzer, ${ }^{34}$ P. Squillacioti, ${ }^{47 c, 47 a}$ M. Stanitzki ${ }^{61}$ R. St. Denis, ${ }^{22}$ B. Stelzer, ${ }^{34}$ O. Stelzer-Chilton, ${ }^{34}$ D. Stentz, ${ }^{39}$ J. Strologas, ${ }^{38}$ G. L. Strycker,${ }^{35}$ D. Stuart, ${ }^{11}$ J. S. Suh, ${ }^{28}$ A. Sukhanov, ${ }^{19}$ I. Suslov, ${ }^{16}$ T. Suzuki, ${ }^{56}$ A. Taffard, ${ }^{25, g}$ R. Takashima, ${ }^{41}$ Y. Takeuchi,${ }^{56}$ R. Tanaka,${ }^{41}$ M. Tecchio, ${ }^{35}$ P. K. Teng, ${ }^{1}$ K. Terashi,${ }^{51}$ J. Thom, ${ }^{18, i}$ A. S. Thompson, ${ }^{22}$ G. A. Thompson, ${ }^{25}$ E. Thomson, ${ }^{46}$ P. Tipton, ${ }^{61}$ P. Ttito-Guzmán, ${ }^{32}$ S. Tkaczyk, ${ }^{18}$ D. Toback, ${ }^{54}$ S. Tokar, ${ }^{15}$ K. Tollefson, ${ }^{36}$ T. Tomura ${ }^{56}$ D. Tonelli, ${ }^{18}$ S. Torre, ${ }^{20}$ D. Torretta, ${ }^{18}$ P. Totaro, ${ }^{55 b, 55 a}$ S. Tourneur, ${ }^{45}$ M. Trovato, ${ }^{47 a}$ S.-Y. Tsai, ${ }^{1} \mathrm{Y}$. Tu, ${ }^{46}$ N. Turini, ${ }^{47 c, 47 a}$ F. Ukegawa, ${ }^{56}$ S. Vallecorsa ${ }^{21}$ N. van Remortel,${ }^{24, c}$ A. Varganov, ${ }^{35}$ E. Vataga, ${ }^{47 d, 47 a}$ F. Vázquez,${ }^{19, m}$ G. Velev, ${ }^{18}$ C. Vellidis, ${ }^{3}$ M. Vidal,${ }^{32}$ R. Vidal,${ }^{18}$ I. Vila, ${ }^{12}$ R. Vilar, ${ }^{12}$ T. Vine,${ }^{31}$ M. Vogel, ${ }^{38}$ I. Volobouev, ${ }^{29, s}$ G. Volpi, ${ }^{47 b, 47 a}$ P. Wagner, ${ }^{46}$ R. G. Wagner, ${ }^{2}$ R. L. Wagner, ${ }^{18}$ W. Wagner, ${ }^{27, v}$ J. Wagner-Kuhr, ${ }^{27}$ T. Wakisaka, ${ }^{42}$ R. Wallny, ${ }^{9}$ S. M. Wang, ${ }^{1}$ A. Warburton, ${ }^{34}$ D. Waters, ${ }^{31}$ M. Weinberger,${ }^{54}$ J. Weinelt, ${ }^{27}$ W. C. Wester III, ${ }^{18}$ B. Whitehouse,${ }^{57}$ D. Whiteson, ${ }^{46, g}$ A. B. Wicklund, ${ }^{2}$ E. Wicklund, ${ }^{18}$ S. Wilbur, ${ }^{14}$ G. Williams, ${ }^{34}$ H. H. Williams,${ }^{46}$ P. Wilson, ${ }^{18}$ B. L. Winer,${ }^{40}$ P. Wittich, ${ }^{18, i}$ S. Wolbers,${ }^{18}$ C. Wolfe, ${ }^{14}$ T. Wright,${ }^{35} \mathrm{X}$. Wu,${ }^{21}$ F. Würthwein,${ }^{10} \mathrm{~S}$. Xie,${ }^{33}$ A. Yagil,${ }^{10} \mathrm{~K}$. Yamamoto, ${ }^{42} \mathrm{~J}$. Yamaoka ${ }^{17}$ U. K. Yang, ${ }^{14, o}$ Y. C. Yang,${ }^{28}$ W. M. Yao, ${ }^{29}$ G. P. Yeh, ${ }^{18}$ J. Yoh, ${ }^{18}$ K. Yorita, ${ }^{58}$ T. Yoshida, ${ }^{42}$ G. B. Yu, ${ }^{50}$ I. Yu, ${ }^{28}$ S. S. Yu,${ }^{18}$ J. C. Yun, ${ }^{18}$ L. Zanello, ${ }^{52 b, 52 a}$ A. Zanetti, ${ }^{55 a}$ X. Zhang, ${ }^{25}$ Y. Zheng, ${ }^{9, e}$ and S. Zucchelli ${ }^{6 b, 6 a}$

(CDF Collaboration)

\footnotetext{
${ }^{1}$ Institute of Physics, Academia Sinica, Taipei, Taiwan 11529, Republic of China

${ }^{2}$ Argonne National Laboratory, Argonne, Illinois 60439, USA

${ }^{3}$ University of Athens, 15771 Athens, Greece

${ }^{4}$ Institut de Fisica d'Altes Energies, Universitat Autonoma de Barcelona, E-08193, Bellaterra (Barcelona), Spain

${ }^{5}$ Baylor University, Waco, Texas 76798, USA

${ }^{6 a}$ Istituto Nazionale di Fisica Nucleare Bologna, I-40127 Bologna, Italy

${ }^{6 \mathrm{~b}}$ University of Bologna, I-40127 Bologna, Italy

${ }^{7}$ Brandeis University, Waltham, Massachusetts 02254, USA

${ }^{8}$ University of California, Davis, Davis, California 95616, USA

${ }^{9}$ University of California, Los Angeles, Los Angeles, California 90024, USA

${ }^{10}$ University of California, San Diego, La Jolla, California 92093, USA

${ }^{11}$ University of California, Santa Barbara, Santa Barbara, California 93106, USA

${ }^{12}$ Instituto de Fisica de Cantabria, CSIC-University of Cantabria, 39005 Santander, Spain

${ }^{13}$ Carnegie Mellon University, Pittsburgh, Pennsylvania 15213, USA

${ }^{14}$ Enrico Fermi Institute, University of Chicago, Chicago, Illinois 60637, USA

${ }^{15}$ Comenius University, 84248 Bratislava, Slovakia; Institute of Experimental Physics, 04001 Kosice, Slovakia

${ }^{16}$ Joint Institute for Nuclear Research, RU-141980 Dubna, Russia

${ }^{17}$ Duke University, Durham, North Carolina 27708, USA

${ }^{18}$ Fermi National Accelerator Laboratory, Batavia, Illinois 60510, USA

${ }^{19}$ University of Florida, Gainesville, Florida 32611, USA

${ }^{20}$ Laboratori Nazionali di Frascati, Istituto Nazionale di Fisica Nucleare, I-00044 Frascati, Italy

${ }^{21}$ University of Geneva, CH-1211 Geneva 4, Switzerland

${ }^{22}$ Glasgow University, Glasgow G12 8QQ, United Kingdom

${ }^{23}$ Harvard University, Cambridge, Massachusetts 02138, USA

${ }^{24}$ Division of High Energy Physics, Department of Physics, University of Helsinki and Helsinki Institute of Physics, FIN-00014, Helsinki, Finland
} 
${ }^{25}$ University of Illinois, Urbana, Illinois 61801, USA

${ }^{26}$ The Johns Hopkins University, Baltimore, Maryland 21218, USA

${ }^{27}$ Institut für Experimentelle Kernphysik, Universität Karlsruhe, 76128 Karlsruhe, Germany

${ }^{28}$ Center for High Energy Physics: Kyungpook National University, Daegu 702-701, Korea; Seoul National University, Seoul 151-742, Korea;

Sungkyunkwan University, Suwon 440-746, Korea;

Korea Institute of Science and Technology Information, Daejeon, 305-806, Korea;

Chonnam National University, Gwangju, 500-757, Korea

${ }^{29}$ Ernest Orlando Lawrence Berkeley National Laboratory, Berkeley, California 94720, USA

${ }^{30}$ University of Liverpool, Liverpool L69 7ZE, United Kingdom

${ }^{31}$ University College London, London WC1E 6BT, United Kingdom

${ }^{32}$ Centro de Investigaciones Energeticas Medioambientales y Tecnologicas, E-28040 Madrid, Spain

${ }^{33}$ Massachusetts Institute of Technology, Cambridge, Massachusetts 02139, USA

${ }^{34}$ Institute of Particle Physics: McGill University, Montréal, Québec, Canada H3A 2T8;

Simon Fraser University, Burnaby, British Columbia, Canada V5A 1S6;

University of Toronto, Toronto, Ontario, Canada M5S 1A7;

and TRIUMF, Vancouver, British Columbia, Canada V6T $2 A 3$

${ }^{35}$ University of Michigan, Ann Arbor, Michigan 48109, USA

${ }^{36}$ Michigan State University, East Lansing, Michigan 48824, USA

${ }^{37}$ Institution for Theoretical and Experimental Physics, ITEP, Moscow 117259, Russia

${ }^{38}$ University of New Mexico, Albuquerque, New Mexico 87131, USA

${ }^{39}$ Northwestern University, Evanston, Illinois 60208, USA

${ }^{40}$ The Ohio State University, Columbus, Ohio 43210, USA

${ }^{41}$ Okayama University, Okayama 700-8530, Japan

${ }^{42}$ Osaka City University, Osaka 588, Japan

${ }^{43}$ University of Oxford, Oxford OX1 3RH, United Kingdom

${ }^{44 a}$ Istituto Nazionale di Fisica Nucleare, Sezione di Padova-Trento, I-35131 Padova, Italy

${ }^{44 \mathrm{~b}}$ University of Padova, I-35131 Padova, Italy

${ }^{45}$ LPNHE, Universite Pierre et Marie Curie/IN2P3-CNRS, UMR7585, Paris, F-75252 France

${ }^{46}$ University of Pennsylvania, Philadelphia, Pennsylvania 19104, USA

${ }^{47 a}$ Istituto Nazionale di Fisica Nucleare Pisa, I-56127 Pisa, Italy

${ }^{47 \mathrm{~b}}$ University of Pisa, I-56127 Pisa, Italy

${ }^{47 c}$ University of Siena, I-56127 Pisa, Italy

${ }^{47 \mathrm{~d}}$ Scuola Normale Superiore, I-56127 Pisa, Italy

${ }^{48}$ University of Pittsburgh, Pittsburgh, Pennsylvania 15260, USA

${ }^{49}$ Purdue University, West Lafayette, Indiana 47907, USA

${ }^{50}$ University of Rochester, Rochester, New York 14627, USA

${ }^{51}$ The Rockefeller University, New York, New York 10021, USA

${ }^{\mathrm{a}}$ Deceased.

${ }^{\mathrm{b}}$ Visitor from University of Massachusetts Amherst, Amherst, MA 01003, USA.

${ }^{\mathrm{c}}$ Visitor from Universiteit Antwerpen, B-2610 Antwerp, Belgium.

${ }^{\mathrm{d}}$ Visitor from University of Bristol, Bristol BS8 1TL, United Kingdom.

${ }^{\mathrm{e}}$ Visitor from Chinese Academy of Sciences, Beijing 100864, China.

${ }^{\mathrm{f}}$ Visitor from Istituto Nazionale di Fisica Nucleare, Sezione di Cagliari, 09042 Monserrato (Cagliari), Italy.

${ }^{\mathrm{g}}$ Visitor from University of California Irvine, Irvine, CA 92697, USA.

${ }^{\mathrm{h}}$ Visitor from University of California Santa Cruz, Santa Cruz, CA 95064, USA.

${ }^{\mathrm{i}}$ Visitor from Cornell University, Ithaca, NY 14853, USA.

${ }^{\mathrm{j}}$ Visitor from University of Cyprus, Nicosia CY-1678, Cyprus.

${ }^{\mathrm{k}}$ Visitor from University College Dublin, Dublin 4, Ireland.

${ }^{1}$ Visitor from University of Edinburgh, Edinburgh EH9 3JZ, United Kingdom.
${ }^{\mathrm{m}}$ Visitor from Universidad Iberoamericana, Mexico D.F., Mexico.

${ }^{\mathrm{n}}$ Visitor from Queen Mary, University of London, London, E1 4NS, England, United Kingdom.

${ }^{\circ}$ Visitor from University of Manchester, Manchester M13 9PL, England, United Kingdom.

${ }^{\mathrm{p}}$ Visitor from Nagasaki Institute of Applied Science, Nagasaki, Japan.

${ }^{\mathrm{q}}$ Visitor from University of Notre Dame, Notre Dame, IN 46556, USA.

${ }^{\mathrm{r}}$ Visitor from University de Oviedo, E-33007 Oviedo, Spain.

${ }^{\mathrm{s}}$ Visitor from Texas Tech University, Lubbock, TX 79409 , USA.

${ }^{t}$ Visitor from IFIC (CSIC-Universitat de Valencia), 46071 Valencia, Spain.

"Visitor from University of Virginia, Charlottesville, VA 22904, USA.

${ }^{\vee}$ Visitor from Bergische Universität Wuppertal, 42097 Wuppertal, Germany.

${ }^{\mathrm{w}}$ On leave from J. Stefan Institute, Ljubljana, Slovenia. 


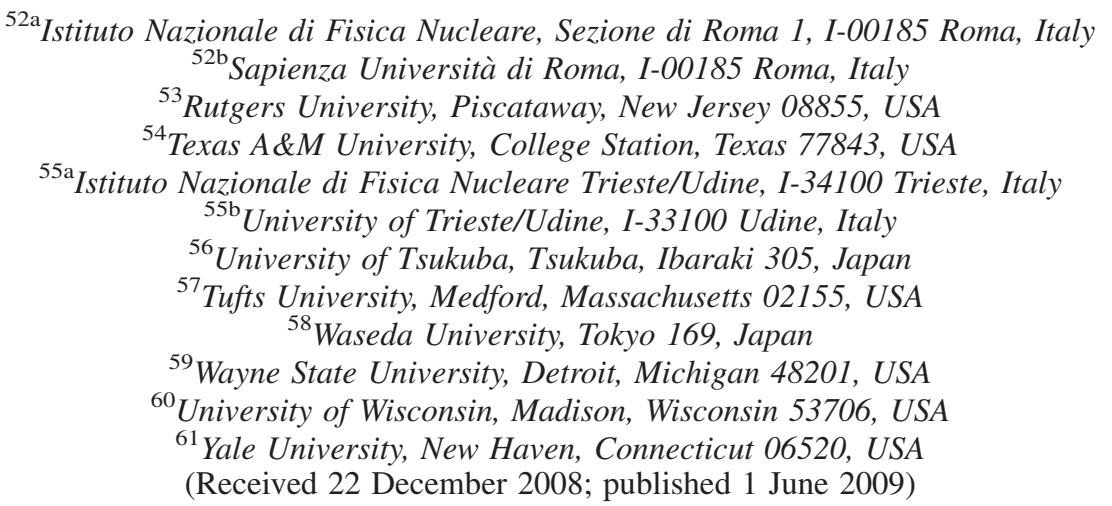

We present a search for new particles which produce narrow two-jet (dijet) resonances using protonantiproton collision data corresponding to an integrated luminosity of $1.13 \mathrm{fb}^{-1}$ collected with the CDF II detector. The measured dijet mass spectrum is found to be consistent with next-to-leading-order perturbative QCD predictions, and no significant evidence of new particles is found. We set upper limits at the $95 \%$ confidence level on cross sections times the branching fraction for the production of new particles decaying into dijets with both jets having a rapidity magnitude $|y|<1$. These limits are used to determine the mass exclusions for the excited quark, axigluon, flavor-universal coloron, $E_{6}$ diquark, coloroctet techni- $\rho, W^{\prime}$, and $Z^{\prime}$.

DOI: 10.1103/PhysRevD.79.112002

PACS numbers: 13.85.Rm, 14.70.Pw, 14.80.-j

\section{INTRODUCTION}

Within the standard model (SM), two-jet (dijet) events are produced in proton-antiproton $(p \bar{p})$ collisions predominantly from hard quantum chromodynamics (QCD) interactions of two partons. The fragmentation and hadronization of the outgoing partons produce hadronic jets. The dijet mass spectrum predicted by QCD falls smoothly and steeply with increasing dijet mass. Many extensions of the SM predict the existence of new massive particles that decay into two energetic partons (quarks, q, or gluons, $g$ ), which can potentially be observed as a narrow resonance in the dijet mass spectrum. Such particles include the excited quark [1], axigluon [2], flavor-universal coloron [3], color-octet techni- $\rho$ [4], Randall-Sundrum (RS) graviton [5], $W^{\prime}, Z^{\prime}$ [6], and diquark in the string-inspired $E_{6}$ model [7].

Here we briefly discuss the theoretical models for these new particles. In the SM, the quarks are considered as fundamental particles. However, the presence of their generational structure and mass hierarchy motivates models of quark compositeness in which the quarks consist of more fundamental particles. If a quark is a composite particle, an excited state of a quark $q^{*}$ is expected, which decays to $q g$ [1]. In chiral color models, the $S U(3)$ gauge group of QCD results from the spontaneous breaking of the chiral color gauge group of $S U(3) \times S U(3)$. Any model of chiral color predicts the presence of the axigluon, a massive axial vector gluon, that decays to $q \bar{q}$ [2]. The flavor-universal coloron model also embeds the $S U(3)$ of QCD in a larger gauge group and predicts the presence of a color-octet coloron which decays to $q \bar{q}$ [3]. Technicolor models seek to explain electroweak symmetry breaking via the dynam- ics of new interactions among techniquarks. The models of extended technicolor and topcolor-assisted technicolor predict the presence of a color-octet techni- $\rho\left(\rho_{T 8}\right)$ which decays to $q \bar{q}$ or $g g$ [4]. The RS model of a warped extra dimension offers a solution for the hierarchy between the electroweak scale and Planck scale $\bar{M}_{\mathrm{Pl}}$ by introducing an extra spacial dimension [5]. This model predicts a KaluzaKlein tower of graviton states (RS gravitons) which decay to $q \bar{q}$ or $g g$. The grand unified theories (GUT) based on larger gauge groups, e.g., $E_{6}$ and $S O(10)$, or left-rightsymmetric models [8] often introduce additional gauge bosons, such as $W^{\prime}$ and $Z^{\prime}$, which decay to $q \bar{q}^{\prime}$ and $q \bar{q}$, respectively [6]. The $E_{6}$ GUT model also predicts the presence of a diquark which decays to $q q$ or $\bar{q} \bar{q}$ [7].

In the past, the UA2 [9], CDF [10,11], and D0 [12] experiments searched for resonances in the dijet mass spectrum and set limits on their production. In this article, we present a first measurement of the dijet mass spectrum and a search for massive particles which produce narrow dijet resonances in $p \bar{p}$ collisions at the center-of-mass energy $\sqrt{s}=1.96 \mathrm{TeV}$. This analysis uses data corresponding to an integrated luminosity of $1.13 \mathrm{fb}^{-1}$ collected between February 2002 and February 2006 with the CDF II detector at the Fermilab Tevatron.

The measurement of the dijet mass spectrum is also an important test of perturbative QCD (pQCD) predictions. It provides complementary information to the inclusive jet cross section measurements [13-17], and comparisons of the measurement with $\mathrm{pQCD}$ predictions provide constraints on the parton distribution functions (PDFs) of the proton, in particular, at high momentum fraction $x(x \geqslant$ 0.3 , where the gluon distribution is not well constrained [18]. 


\section{THE CDF DETECTOR}

The CDF II detector is described in detail elsewhere [19]. Here, the components that are relevant to this search are briefly described. Surrounding the beam pipe, there is a tracking system consisting of a silicon microstrip detector, a cylindrical drift chamber, and a solenoid magnet that provides a $1.4 \mathrm{~T}$ magnetic field. The central and plug calorimeters, which cover the pseudorapidity regions of $|\eta|<1.1$ and $1.1<|\eta|<3.6$ [20], respectively, surround the tracking system with a projective tower geometry and measure the energy of interacting particles. The calorimeters are segmented into electromagnetic and hadronic sections. The electromagnetic section consists of lead and scintillator, and the hadronic section consists of iron and scintillator, respectively. In the central region, the calorimeter consists of 48 modules, segmented into towers of granularity $\Delta \eta \times \Delta \phi \approx 0.1 \times 0.26$. The energy resolution of the central electromagnetic calorimeter for electrons is $\sigma\left(E_{T}\right) / E_{T}=13.5 \% / \sqrt{E_{T}(\mathrm{GeV})} \oplus 1.5 \%$, while the energy resolution of the central hadron calorimeter for charged pions that do not interact in the electromagnetic section is $\sigma\left(E_{T}\right) / E_{T}=50 \% / \sqrt{E_{T}(\mathrm{GeV})} \oplus 3 \%$, where $E_{T}$ is the transverse energy [20]. The wall hadron calorimeter covers the gap in the projective tower geometry between the central and plug hadron calorimeters, corresponding to $0.7<|\eta|<1.3$, with segmentation similar to that of the central calorimeter. The energy resolution of the wall hadron calorimeter is $\sigma\left(E_{T}\right) / E_{T}=75 \% / \sqrt{E_{T}(\mathrm{GeV})} \oplus$ $4 \%$ for charged pions that do not interact in the electromagnetic section. A system of Cherenkov counters, located around the beam pipe and inside the plug calorimeters, is used to measure the number of inelastic $p \bar{p}$ collisions per bunch crossing and thereby the luminosity.

\section{DATA SETS AND EVENT SELECTION}

The dijet data used in this search were collected using a three-level on-line event selection (trigger) system, and are identical to the data used in Ref. [17]. The four trigger selections used in this analysis are referred to as "jet20," "jet50," "jet70," and "jet100" according to the $E_{T}$ threshold (in $\mathrm{GeV}$ ) of calorimeter clusters reconstructed using a cone algorithm [21] with cone radius $R_{\text {cone }}=0.7$. The jet20, jet50, and jet70 trigger rates are randomly reduced (prescaled) to avoid saturating the bandwidth of the data acquisition system. After prescaling, these trigger data sets correspond to $1.44,32.5$, and $143 \mathrm{pb}^{-1}$ of integrated luminosity, respectively. The jet100 trigger is not prescaled.

Jets are reconstructed from the energy depositions in the calorimeter towers with the transverse momentum $p_{T}$ [20] above $0.1 \mathrm{GeV} / c$. Jets are formed from the four-vectors of calorimeter towers [22] using the cone-based midpoint jet clustering algorithm $[14,23,24]$ with cone radius $R_{\text {cone }}=$ 0.7 . The kinematics of a jet is defined by the four-vector recombination scheme [23].
Cosmic ray and beam loss background events are removed by requiring $\mathbb{E}_{T} / \sqrt{\sum E_{T}}<\min (3+0.0125 \times$ $\left.p_{T}^{\text {jet1 }}, 6\right)$, where $\mathscr{E}_{T}$ and $\sum E_{T}$ are the missing $E_{T}$ and sum $E_{T}$ [25], respectively, and $p_{T}^{\text {jetl }}(\mathrm{GeV} / c)$ is the $p_{T}$ of the leading jet in the event before the corrections described in Sec. IV are applied. This requirement makes the fraction of background events negligible and has an efficiency of $\gtrsim 95 \%$ for dijet events. In order to ensure good coverage of each event by the detectors, the primary event vertex is required to be within $60 \mathrm{~cm}$ of the center of the detector along the $z$ axis [20]. The efficiency for this requirement is determined to be $96 \%$ from the distribution of primary event vertices along $z$ measured using a sample of minimum bias events.

\section{JET CORRECTIONS AND MEASUREMENT OF THE DIJET MASS SPECTRUM}

The jet energies measured by the calorimeters are affected by instrumental effects such as calorimeter nonuniformity, nonlinearity, and energy smearing. We correct for these biases in several steps [26]. First, an $\eta$-dependent relative correction is applied to equalize the response of the calorimeter. The equalized jet $p_{T}$ is then corrected for the effects of pileup, i.e., additional $p \bar{p}$ interactions in the same bunch crossing. The pileup correction subtracts $0.97 \pm 0.29 \mathrm{GeV} / c$ for each additional primary vertex from the measured jet $p_{T}$. Then, a $p_{T}$-dependent correction is applied to account for, on average, the undermeasured hadron energy due to the nonlinearity of the calorimeter response. The correction factors are 1.19 and 1.06 at jet $p_{T}=90$ and $600 \mathrm{GeV} / c$, respectively. After these corrections we reconstruct the dijet mass $m_{j j}$ from the fourvectors of the two highest corrected- $p_{T}$ jets. We form the dijet mass spectrum as

$$
\left.\frac{d \sigma}{d m_{j j}}\right|_{i}=\frac{U_{i}}{\mathcal{L}_{i} \cdot \epsilon_{\text {trig }, i}} \frac{n_{i}}{\Delta m_{j j, i}}
$$

where $n_{i}$ is the observed number of events, $\epsilon_{\text {trig, } i}$ is the trigger efficiency, $U_{i}$ is the unfolding correction, $\Delta m_{j j, i}$ is the bin width, and $\mathcal{L}_{i}$ is the integrated luminosity of the trigger data set used for the $i$ th dijet mass bin. The unfolding correction accounts for the bin-by-bin migration effect due to the finite resolution of the $m_{j j}$ measurement and the efficiencies of the off-line event selection requirements, and is discussed in detail below. The bin width $m_{j j}$ is set to $10 \%$ of the dijet mass which approximately corresponds to the dijet mass resolution. We count only events in which both of the leading two jets have a rapidity magnitude $|y|$ [20] less than 1.

We use the jet20, jet50, jet70, and jet100 data sets for the dijet mass regions of 180-241, 241-321, 321-427, and above $427 \mathrm{GeV} / c^{2}$, respectively, where the trigger efficiencies are higher than $99.8 \%$. This ensures a negligible uncertainty from trigger efficiency measurements. The 
relative difference in the integrated luminosity between jet70 and jet100 is determined by taking the ratio of jet70 to jet100 data in the dijet mass region where both triggers are fully efficient, and the relative differences between the jet50 and jet70, and jet 20 and jet50 integrated luminosities are determined in the same manner. This ensures no bias at the dijet mass boundaries between different trigger data sets.

The measured spectrum is corrected for the bin-by-bin migration effect due to the finite resolution of the $m_{j j}$ measurement and the efficiencies of the off-line event selection requirements by the unfolding correction $U_{i}$ when it is compared with QCD predictions at the hadron level [17]. We obtain the unfolding correction using QCD dijet events generated by the PYTHIA 6.2 [27] Monte Carlo simulation program that have passed through the $\mathrm{CDF}$ detector simulation [28]. The PYTHIA events are generated with Tune A [29], which refers to the set of parameters describing multiple-parton interactions and initial state radiation that have been tuned to reproduce the energy observed in the region transverse to the leading jet [30]. It has also been shown to provide a reasonable description of the measured energy distribution inside a jet [31]. The correction is determined on a bin-by-bin basis by taking the ratio of a hadron-level cross section to a calorimeter-level cross section. The hadron-level cross section is defined using hadron-level jets clustered from the final state stable particles [32] in PYTHIA with the same jet clustering algorithm as the one used to cluster calorimeter towers. The leading two jets are required to have $|y|<1$. The calorimeter-level cross section is obtained by analyzing the PYTHIA events using the same analysis chain as for the data. Since the correction depends on the dijet mass spectrum, the PYTHIA events are reweighted to match the dijet mass spectrum measured in data before the correction factor is calculated. The size of the correction ranges from $\sim 1.2$ at low $m_{j j}\left(200 \mathrm{GeV} / c^{2}\right)$ to $\sim 1.5$ at high $m_{j j}$ $\left(1250 \mathrm{GeV} / c^{2}\right)$.

The systematic uncertainties arise mainly from four sources: the jet energy scale, the jet energy resolution, the unfolding correction, and the integrated luminosity. The dominant source is from the absolute jet energy scale. The size of the uncertainty in the cross section varies from $10 \%$ at low $m_{j j}$ to ${ }_{-47}^{+74} \%$ at high $m_{j j}$. The uncertainty in the relative jet energy scale introduces a $3 \%$ uncertainty on the measured cross section at low $m_{j j}$ and ${ }_{-9}^{+10} \%$ at high $m_{j j}$. The uncertainty in the modeling of jet energy smearing is estimated from the difference between the data and PYTHIA samples using the bisector method [33], and it introduces an uncertainty in the cross section of $1 \%$ at low $m_{j j}$ and ${ }_{-5}^{+6} \%$ at high $m_{j j}$. The difference between the unfolding correction from a PYTHIA sample and that from a sample generated by the HERWIG 6.5 [34] Monte Carlo simulation program is also taken as a systematic uncertainty to account for the uncertainty in the modeling of jet fragmenta-

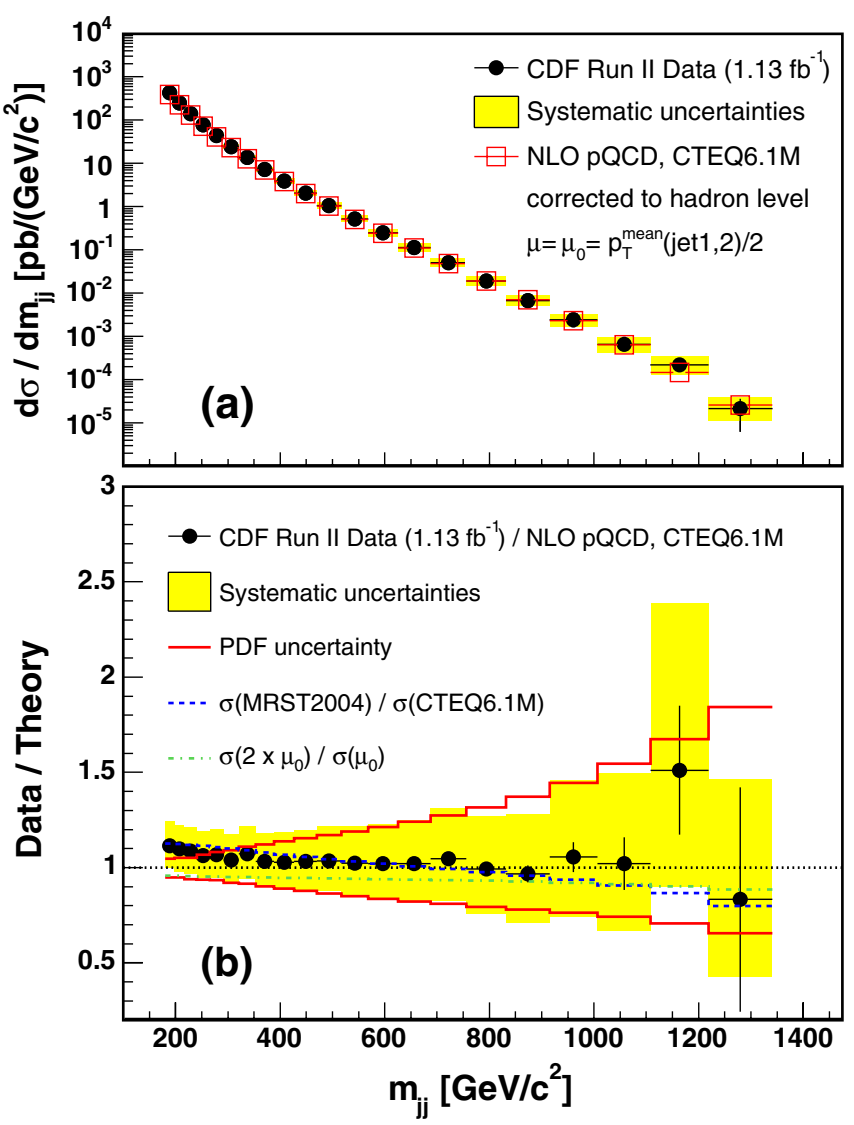

FIG. 1 (color online). (a) The measured dijet mass spectrum for both jets to have $|y|<1$ compared to the NLO pQCD prediction obtained using the CTEQ6.1 PDFs. (b) The ratio of the data to the NLO pQCD prediction. The experimental systematic uncertainties, theoretical uncertainties from PDF, the ratio of MRST2004/CTEQ6.1, and the dependence on the choice of renormalization and factorization scales are also shown. An additional $6 \%$ uncertainty in the determination of the luminosity is not shown.

tion. This uncertainty is $2 \%$ at low $m_{j j}$ and $8 \%$ at high $m_{j j}$. The uncertainty in the determination of the integrated luminosity is $6 \%$, independent of $m_{j j}$. The total uncertainty is ${ }_{-12}^{+13} \%$ at low $m_{j j}$ and ${ }_{-49}^{+76} \%$ at high $m_{j j}$. The measured dijet mass spectrum after all the corrections discussed above are applied is shown together with the statistical and systematic uncertainties in Fig. 1(a).

\section{COMPARISONS WITH QCD PREDICTIONS}

The measured dijet mass spectrum is compared in Fig. 1 to the next-to-leading-order (NLO) pQCD predictions from FASTNLO [35]. The predictions were obtained using the CTEQ6.1 [18] PDFs with the renormalization and factorization scales both set to $\mu_{0}$, the average $p_{T}$ of the leading two jets. Jets are reconstructed by the midpoint algorithm with cone radius $R_{\text {cone }}=0.7$. The maximum separation between two partons merged into a jet is set to $R_{\text {cone }} \times R_{\text {sep }}$ [36], and $R_{\text {sep }}$ is set to $1.3[21,24]$. Setting the 
renormalization and factorization scales to $2 \mu_{0}$ instead of $\mu_{0}$ reduces the cross section prediction by $5 \%-10 \%$, and setting $R_{\text {sep }}=2$ increases the cross section by $\lesssim 10 \%$. The PDF uncertainties estimated from 40 CTEQ6.1 error PDFs and the ratio of the predictions using MRST2004 [37] and CTEQ6.1 are shown in Fig. 1(b). The PDF uncertainty is the dominant theoretical uncertainty for most of the $m_{j j}$ range. The NLO pQCD predictions for jets clustered from partons need to be corrected for nonperturbative underlying event and hadronization effects. The multiplicative parton-to-hadron-level correction $\left(C_{p \rightarrow h}\right)$ is determined on a bin-by-bin basis from a ratio of two dijet mass spectra. The numerator is the nominal hadron-level dijet mass spectrum from the PYTHIA Tune A samples, and the denominator is the dijet mass spectrum obtained from jets formed from partons before hadronization in a sample simulated with an underlying event turned off. We assign the difference between the corrections obtained using HERWIG and PYTHIA Tune A as the uncertainty on the $C_{p \rightarrow h}$ correction. The $C_{p \rightarrow h}$ correction is $1.16 \pm 0.08$ at low $m_{j j}$ and $1.02 \pm 0.02$ at high $m_{j j}$. Figure 1 shows the ratio of the measured spectrum to the NLO $\mathrm{pQCD}$ predictions corrected for the nonperturbative effects. The data and theoretical predictions are found to be in good agreement. To quantify the agreement, we performed a $\chi^{2}$ test which is the same as the one used in the inclusive jet cross section measurements $[15,17]$. The test treats the systematic uncertainties from different sources and uncertainties on $C_{p \rightarrow h}$ as independent but fully correlated over all $m_{j j}$ bins and yields $\chi^{2} /$ no. d.o.f. $=21 / 21$.

\section{SEARCH FOR DIJET MASS RESONANCES}

We search for narrow mass resonances in the measured dijet mass spectrum by fitting the measured spectrum to a smooth functional form and by looking for data points that show significant excess from the fit. We fit the measured dijet mass spectrum before the bin-by-bin unfolding correction is applied. We use the following functional form:

$$
\frac{d \sigma}{d m_{j j}}=p_{0}(1-x)^{p_{1}} / x^{p_{2}+p_{3} \cdot \ln (x)}, \quad x=m_{j j} / \sqrt{s},
$$

where $p_{0}, p_{1}, p_{2}$, and $p_{3}$ are free parameters. This form fits well the dijet mass spectra from PYTHIA, HERWIG, and NLO pQCD predictions. The result of the fit to the measured dijet mass spectrum is shown in Fig. 2. Equation (2) fits the measured dijet mass spectrum well with $\chi^{2} /$ no. d.o.f. $=$ $16 / 17$. We find no evidence for the existence of a resonant structure, and in the next section we use the data to set limits on new particle production.

\section{LIMITS ON NEW PARTICLE PRODUCTION}

Several theoretical models which predict the existence of new particles that produce narrow dijet resonances are considered in this search. For the excited quark $q^{*}$ which

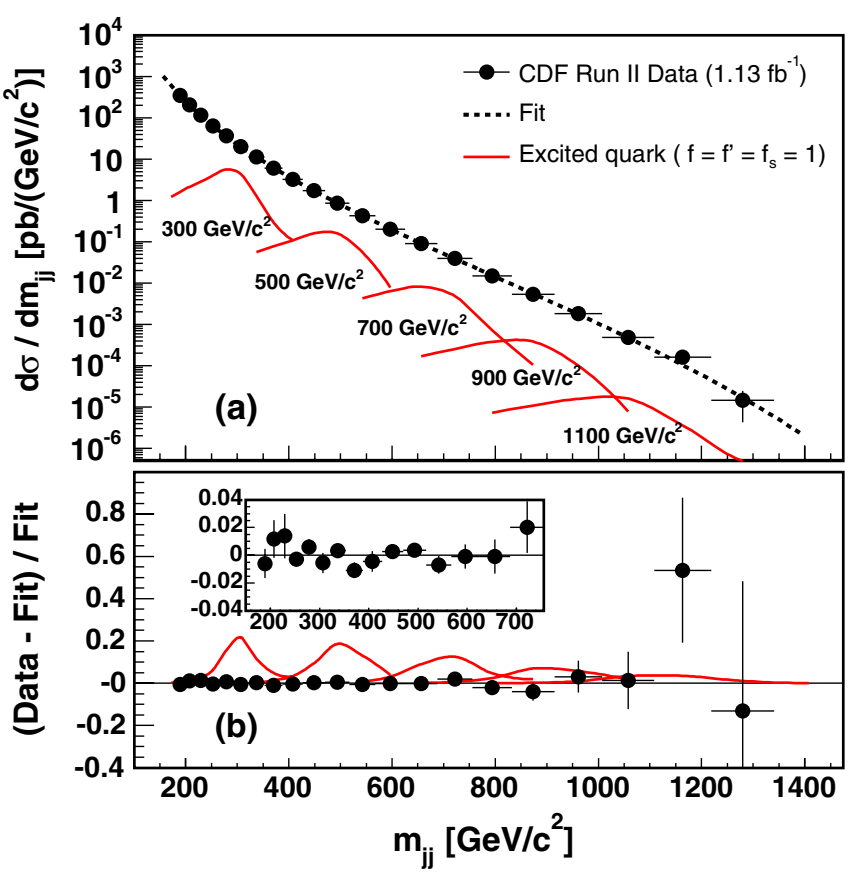

FIG. 2 (color online). (a) The measured dijet mass spectrum (points) fitted to Eq. (2) (dashed curve). The bin-by-bin unfolding corrections is not applied. Also shown are the predictions from the excited quark, $q^{*}$, simulations for masses of 300,500 , 700,900 , and $1100 \mathrm{GeV} / c^{2}$, respectively (solid curves). (b) The fractional difference between the measured dijet mass distribution and the fit (points) compared to the predictions for $q^{*}$ signals divided by the fit to the measured dijet mass spectrum (curves). The inset shows the expanded view in which the vertical scale is restricted to \pm 0.04 .

decays to $q g$, we set its couplings to the SM $S U(2), U(1)$, and $S U(3)$ gauge groups to be $f=f^{\prime}=f_{s}=1$ [1], respectively, and the compositeness scale to the mass of $q^{*}$. For the RS graviton $G^{*}$ that decays into $q \bar{q}$ or $g g$, we use the model parameter $k / \bar{M}_{\mathrm{Pl}}=0.1$ which determines the couplings of the graviton to the SM particles. The production cross section increases with increasing $k / \bar{M}_{\mathrm{Pl}}$; however, values of $k / \bar{M}_{\mathrm{Pl}} \gg 0.1$ are disfavored theoretically [38]. For $W^{\prime}$ and $Z^{\prime}$, which decay to $q \bar{q}^{\prime}$ and $q \bar{q}$ respectively, we use the SM couplings. The leading-order production cross sections of the RS graviton, $W^{\prime}$, and $Z^{\prime}$ are multiplied by a factor of 1.3 to account for higher-order effects in the strong coupling constant $\alpha_{s}$ [39]. All these models are simulated with PYTHIA Tune A. Signal events of these models from PYTHIA are then passed through the CDF detector simulation. For all the models considered in this search, new particle decays into the modes containing the top quark are neither included in the $\sigma^{\text {sig }}$ predictions nor in the signal dijet mass distribution modeling, since such decays generally do not lead to the dijet topology.

The dijet mass distributions from $q^{*}$ simulations with masses $300,500,700,900$, and $1100 \mathrm{GeV} / c^{2}$ are shown in Fig. 2. The dijet mass distributions for the $q^{*}$, RS graviton, 


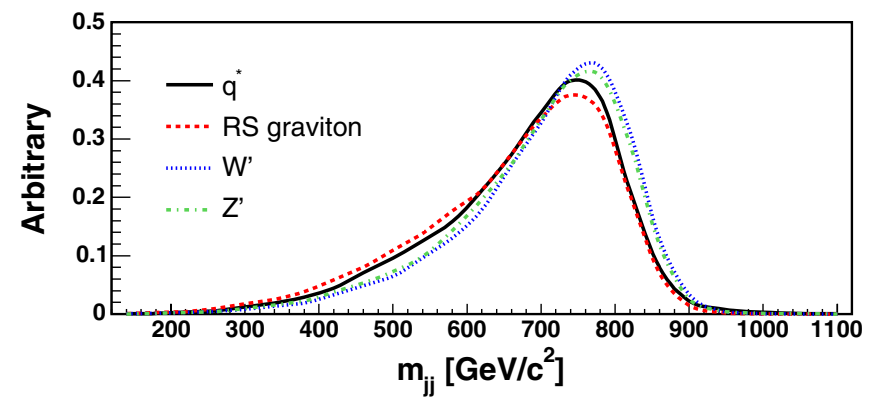

FIG. 3 (color online). Dijet mass distributions for simulated signals of the $q^{*}$, RS graviton, $W^{\prime}$, and $Z^{\prime}$ with the mass of $800 \mathrm{GeV} / c^{2}$.

$W^{\prime}$, and $Z^{\prime}$ simulations with the mass of $800 \mathrm{GeV} / c^{2}$ are shown together in Fig. 3. The shapes of the distributions are mainly determined by the jet energy resolution and QCD radiation which leads to tails on the low mass side. Since the natural width of these particles is substantially smaller than the width from the jet energy resolution, all the dijet mass distributions appear similar. However, the dijet mass resonance distributions are somewhat broader for $q^{*}$ and RS gravitons than for $W^{\prime}$ and $Z^{\prime}$ because $q^{*}$ and RS gravitons can decay into the mode containing gluons, unlike $W^{\prime}$ and $Z^{\prime}$. Gluons radiate more than quarks and tend to make the resulting dijet mass distributions broader. As a result, the cross section limits obtained based on the $q^{*}$ and RS graviton resonance shapes are about 20\% larger than those obtained with the $W^{\prime}$ and $Z^{\prime}$ resonance shapes.

We also consider production of the axigluon $A$ that decays into $q \bar{q}, E_{6}$ diquark $D\left(D^{c}\right)$ that decays into $\bar{q} \bar{q}$ $(q q)$, and color-octet techni- $\rho\left(\rho_{T 8}\right)$ that decays into $q \bar{q}$ or $g g$. Their lowest-order theoretical predictions for $\sigma^{\text {sig }} \equiv$ $\sigma \cdot \mathcal{B} \cdot \mathcal{A}$ are shown in Fig. 4 along with the predictions for the other models described above as a function of new particle mass, where $\sigma$ is the new particle production cross section, $\mathcal{B}$ is the branching fraction to dijets, and $\mathcal{A}$ is the kinematical acceptance for each of the leading two jets to have $|y|<1$. In addition, the flavor-universal coloron $C$ which decays to $q \bar{q}$ is considered. The cross section for the coloron is always larger than or equal to that for the axigluon, so the limits on the axigluon apply to the coloron as well. For $\rho_{T 8}$ production, predictions are for the massdegenerate $\rho_{T 8}$ with the standard topcolor-assistedtechnicolor couplings and with the set of parameters in [40].

We set upper limits on $\sigma^{\mathrm{sig}} \equiv \sigma \cdot \mathcal{B} \cdot \mathcal{A}$ as follows. We use the likelihood function $L=\prod_{i} \mu_{i}^{n_{i}} \exp \left(-\mu_{i}\right) / n_{i}$ !, where $\mu_{i} \equiv \sigma^{\text {sig }} \mathcal{L}_{i} \epsilon_{i} n_{i}^{\text {sig }} / n_{\text {tot }}^{\text {sig }}+n_{i}^{\mathrm{QCD}}$ is the predicted number of events, $\epsilon_{i}$ is the event selection efficiency in the $i$ th dijet mass bin, and $n_{i}^{\mathrm{sig}} / n_{\text {tot }}^{\mathrm{sig}}$ is the predicted fraction of signal events in bin $i$. We model the QCD dijet mass spectrum with Eq. (2) and extract $n_{i}^{\mathrm{QCD}}$ by $n_{i}^{\mathrm{QCD}}=\mathcal{L}_{i}$. $\epsilon_{\text {trig, }, i} \cdot \Delta m_{j j} \cdot d \sigma /\left.d m_{j j}\right|_{i}$. For each value of $\sigma^{\text {sig }}$ we maximize the likelihood with respect to the four parameters in

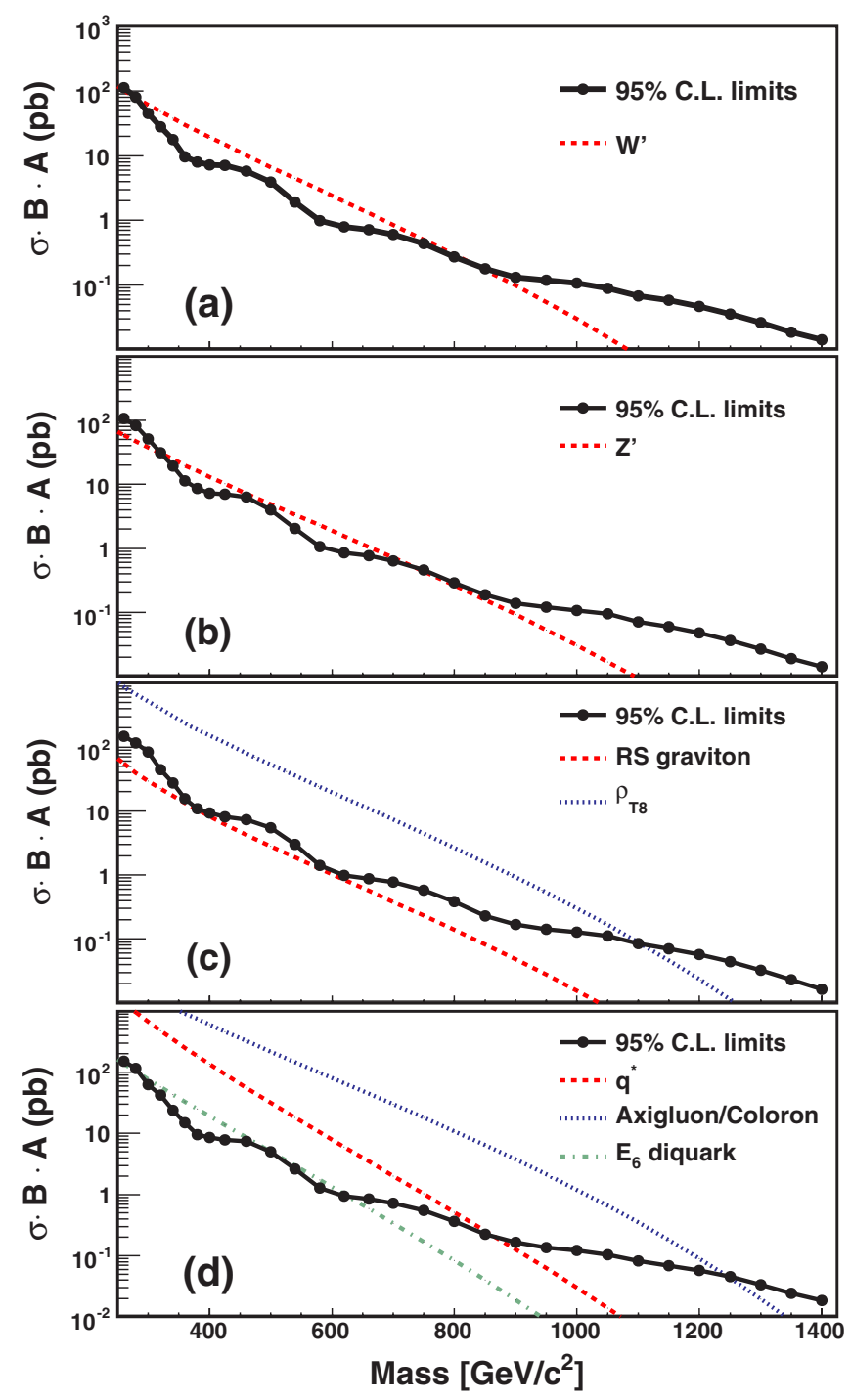

FIG. 4 (color online). Observed 95\% C.L. upper limits on new particle production cross sections times the branching fraction to dijets obtained with the signal shapes from (a) $W^{\prime}$, (b) $Z^{\prime}$, (c) RS graviton, and (d) $q^{*}$ production. Also shown are the cross section predictions for the production of $W^{\prime}, Z^{\prime}$, RS graviton, $\rho_{T 8}, q^{*}$, axigluon, flavor-universal coloron, and $E_{6}$ diquark for the set of parameters described in the text. The limits and theoretical predictions are for events in which both of the leading two jets have $|y|<1$.

Eq. (2). We integrate this profiled likelihood over Bayesian priors for the parameters describing the systematic uncertainties [41], and we use a flat prior on $\sigma^{\text {sig }}$ to extract Bayesian upper limits on that parameter. Although this procedure uses Bayesian techniques, we verified that the resulting upper limits have good frequentist coverage.

The obtained $95 \%$ confidence level (C.L.) limits using the $W^{\prime}, Z^{\prime}$, RS graviton, and $q^{*}$ signal resonance shapes are shown in Fig. 4 and Table I as a function of the new particle mass. Also shown in Fig. 4 are the theoretical predictions for the various models. For the $W^{\prime}, Z^{\prime}, q^{*}$, and RS graviton, 
TABLE I. Observed 95\% C.L. upper limits on the new particle production cross sections times branching fraction to dijets times the acceptance for both jets to have $|y|<1$ obtained with the signal shapes from $W^{\prime}, Z^{\prime}$, RS graviton $\left(G^{*}\right)$, and $q^{*}$ production.

\begin{tabular}{|c|c|c|c|c|}
\hline \multirow{2}{*}{$\begin{array}{c}\text { Mass } \\
\left(\mathrm{GeV} / c^{2}\right)\end{array}$} & \multicolumn{4}{|c|}{ 95\% C.L. $\sigma \cdot \mathcal{B} \cdot \mathcal{A}(\mathrm{pb})$} \\
\hline & $W^{\prime}$ & $Z^{\prime}$ & $G^{*}$ & $q^{*}$ \\
\hline 260 & $1.1 \times 10^{2}$ & $1.1 \times 10^{2}$ & $1.5 \times 10^{2}$ & $1.5 \times 10^{2}$ \\
\hline 280 & $8.1 \times 10^{1}$ & $8.3 \times 10^{1}$ & $1.2 \times 10^{2}$ & $1.1 \times 10^{2}$ \\
\hline 300 & $4.5 \times 10^{1}$ & $5.1 \times 10^{1}$ & $8.3 \times 10^{1}$ & $6.3 \times 10^{1}$ \\
\hline 320 & $2.8 \times 10^{1}$ & $3.1 \times 10^{1}$ & $4.4 \times 10^{1}$ & $4.2 \times 10^{1}$ \\
\hline 340 & $1.8 \times 10^{1}$ & $1.9 \times 10^{1}$ & $2.8 \times 10^{1}$ & $2.4 \times 10^{1}$ \\
\hline 360 & $1.0 \times 10^{1}$ & $1.1 \times 10^{1}$ & $1.6 \times 10^{1}$ & $1.5 \times 10^{1}$ \\
\hline 380 & $8.0 \times 10^{0}$ & $8.6 \times 10^{0}$ & $1.1 \times 10^{1}$ & $1.0 \times 10^{1}$ \\
\hline 400 & $7.2 \times 10^{0}$ & $7.3 \times 10^{0}$ & $9.2 \times 10^{0}$ & $8.6 \times 10^{0}$ \\
\hline 425 & $7.1 \times 10^{0}$ & $7.0 \times 10^{0}$ & $8.2 \times 10^{0}$ & $7.8 \times 10^{0}$ \\
\hline 460 & $5.8 \times 10^{0}$ & $6.3 \times 10^{0}$ & $7.4 \times 10^{0}$ & $7.4 \times 10^{0}$ \\
\hline 500 & $3.9 \times 10^{0}$ & $4.0 \times 10^{0}$ & $5.5 \times 10^{0}$ & $5.0 \times 10^{0}$ \\
\hline 540 & $1.9 \times 10^{0}$ & $2.0 \times 10^{0}$ & $3.0 \times 10^{0}$ & $2.6 \times 10^{0}$ \\
\hline 580 & $1.0 \times 10^{0}$ & $1.1 \times 10^{0}$ & $1.4 \times 10^{0}$ & $1.3 \times 10^{0}$ \\
\hline 620 & $8.0 \times 10^{-1}$ & $8.5 \times 10^{-1}$ & $1.0 \times 10^{0}$ & $1.0 \times 10^{0}$ \\
\hline 660 & $7.2 \times 10^{-1}$ & $7.6 \times 10^{-1}$ & $8.8 \times 10^{-1}$ & $8.4 \times 10^{-1}$ \\
\hline 700 & $6.0 \times 10^{-1}$ & $6.4 \times 10^{-1}$ & $7.8 \times 10^{-1}$ & $7.3 \times 10^{-1}$ \\
\hline 750 & $4.3 \times 10^{-1}$ & $4.6 \times 10^{-1}$ & $5.7 \times 10^{-1}$ & $5.6 \times 10^{-1}$ \\
\hline 800 & $2.7 \times 10^{-1}$ & $2.9 \times 10^{-1}$ & $3.9 \times 10^{-1}$ & $3.7 \times 10^{-1}$ \\
\hline 850 & $1.8 \times 10^{-1}$ & $1.9 \times 10^{-1}$ & $2.3 \times 10^{-1}$ & $2.3 \times 10^{-1}$ \\
\hline 900 & $1.3 \times 10^{-1}$ & $1.4 \times 10^{-1}$ & $1.7 \times 10^{-1}$ & $1.6 \times 10^{-1}$ \\
\hline 950 & $1.2 \times 10^{-1}$ & $1.2 \times 10^{-1}$ & $1.4 \times 10^{-1}$ & $1.4 \times 10^{-1}$ \\
\hline 1000 & $1.1 \times 10^{-1}$ & $1.1 \times 10^{-1}$ & $1.3 \times 10^{-1}$ & $1.2 \times 10^{-1}$ \\
\hline 1050 & $8.9 \times 10^{-2}$ & $9.4 \times 10^{-2}$ & $1.1 \times 10^{-1}$ & $1.0 \times 10^{-1}$ \\
\hline 1100 & $6.7 \times 10^{-2}$ & $7.1 \times 10^{-2}$ & $8.5 \times 10^{-2}$ & $8.2 \times 10^{-2}$ \\
\hline 1150 & $5.8 \times 10^{-2}$ & $5.9 \times 10^{-2}$ & $7.1 \times 10^{-2}$ & $6.9 \times 10^{-2}$ \\
\hline 1200 & $4.6 \times 10^{-2}$ & $4.8 \times 10^{-2}$ & $5.8 \times 10^{-2}$ & $5.7 \times 10^{-2}$ \\
\hline 1250 & $3.6 \times 10^{-2}$ & $3.6 \times 10^{-2}$ & $4.4 \times 10^{-2}$ & $4.5 \times 10^{-2}$ \\
\hline 1300 & $2.6 \times 10^{-2}$ & $2.7 \times 10^{-2}$ & $3.3 \times 10^{-2}$ & $3.4 \times 10^{-2}$ \\
\hline 1350 & $1.8 \times 10^{-2}$ & $1.9 \times 10^{-2}$ & $2.3 \times 10^{-2}$ & $2.4 \times 10^{-2}$ \\
\hline 1400 & $1.4 \times 10^{-2}$ & $1.4 \times 10^{-2}$ & $1.6 \times 10^{-2}$ & $1.8 \times 10^{-2}$ \\
\hline
\end{tabular}

the mass exclusion is determined by comparing the limits obtained with their respective signal shapes and $\sigma^{\text {sig }}$ predictions. For the axigluon, flavor-universal coloron, and $E_{6}$ diquark, we compare their $\sigma^{\text {sig }}$ predictions to the limits obtained with the $q^{*}$ signal shapes; these particles do not decay into the mode containing a gluon, so their signal shape would be narrower than that of $q^{*}$, and thus the mass exclusions obtained with the $q^{*}$ signal shapes are conservative. For $\rho_{T 8}$, we compare its $\sigma^{\text {sig }}$ predictions with the limits obtained using the RS graviton signal shapes. The $\rho_{T 8}$ and RS graviton decay channels are similar; the branching fraction to the $g g$ state is higher for the RS gravitons than for $\rho_{T 8}$, so comparing the limits obtained using the RS graviton signal shapes to the $\rho_{T 8}$ 's $\sigma^{\text {sig }}$ predictions yields a conservative mass exclusion.

The mass exclusion regions obtained in this search are $260<m<870 \mathrm{GeV} / c^{2}$ for $q^{*}, 260<m<1250 \mathrm{GeV} / c^{2}$ for the axigluon and flavor-universal coloron, $290<m<$
$630 \mathrm{GeV} / c^{2}$ for the $E_{6}$ diquark, $260<m<1100 \mathrm{GeV} / c^{2}$ for $\rho_{T 8}, 280<m<840 \mathrm{GeV} / c^{2}$ for $W^{\prime}$, and $320<m<$ $740 \mathrm{GeV} / c^{2}$ for $Z^{\prime}$. For the RS graviton, this search did not exclude any mass region. As the mass exclusions from the previous dijet mass resonance searches [10-12] are $m<$ $775 \mathrm{GeV} / c^{2}$ for $q^{*}, m<980 \mathrm{GeV} / c^{2}$ for the axigluon and flavor-universal coloron, $290<m<420 \mathrm{GeV} / c^{2}$ for the $E_{6}$ diquark, and $260<m<480 \mathrm{GeV} / c^{2}$ for $\rho_{T 8}$, this search provides the most stringent lower mass limits for these particles. This search also extends the existing mass exclusions of $300<m<800 \mathrm{GeV} / c^{2}$ for $W^{\prime}$ and $400<$ $m<640 \mathrm{GeV} / c^{2}$ for $Z^{\prime}$ obtained in the previous dijet mass resonance search [12]. However, the more restrictive mass exclusions of $m<1000 \mathrm{GeV} / c^{2}$ for $W^{\prime}$ and $m<$ $923 \mathrm{GeV} / c^{2}$ for $Z^{\prime}$ come from the $W^{\prime} \rightarrow e \nu$ search [42] and $Z^{\prime} \rightarrow e^{+} e^{-}$search [43].

\section{CONCLUSIONS}

In conclusion, we performed a search for new particles which produce narrow dijet resonances using $p \bar{p}$ collision data from $1.13 \mathrm{fb}^{-1}$ of integrated luminosity collected with the CDF II detector. The measured dijet mass spectrum is found to be consistent with NLO pQCD predictions based on recent PDFs and does not show evidence of a mass resonance from new particle production. We set $95 \%$ confidence level upper limits on new particle production cross sections times the branching fraction to dijets times the acceptance for both jets to have $|y|<1$. We also determine the mass exclusions for the excited quark, axigluon, flavoruniversal coloron, $E_{6}$ diquark, color-octet techni- $\rho, W^{\prime}$, and $Z^{\prime}$ for a specific representative set of model parameters. This search sets the most stringent lower mass limits on the excited quark, the axigluon, the flavor-universal coloron, the $E_{6}$ diquark, and the color-octet techni- $\rho$.

\section{ACKNOWLEDGMENTS}

We thank the Fermilab staff and the technical staffs of the participating institutions for their vital contributions. This work was supported by the U.S. Department of Energy and National Science Foundation; the Italian Istituto Nazionale di Fisica Nucleare; the Ministry of Education, Culture, Sports, Science and Technology of Japan; the Natural Sciences and Engineering Research Council of Canada; the National Science Council of the Republic of China; the Swiss National Science Foundation; the A.P. Sloan Foundation; the Bundesministerium für Bildung und Forschung, Germany; the Korean Science and Engineering Foundation and the Korean Research Foundation; the Science and Technology Facilities Council and the Royal Society, UK; the Institut National de Physique Nucleaire et Physique des Particules/CNRS; the Russian Foundation for Basic Research; the Ministerio de Ciencia e Innovación, and Programa Consolider-Ingenio 2010, Spain; the Slovak R\&D Agency; and the Academy of Finland. 
[1] U. Baur, I. Hinchliffe, and D. Zeppenfeld, Int. J. Mod. Phys. A 2, 1285 (1987); U. Baur, M. Spira, and P. M. Zerwas, Phys. Rev. D 42, 815 (1990).

[2] P. H. Frampton and S. L. Glashow, Phys. Lett. B 190, 157 (1987); J. Bagger, C. Schmidt, and S. King, Phys. Rev. D 37, 1188 (1988).

[3] R. S. Chivukula, A. G. Cohen, and E. H. Simmons, Phys. Lett. B 380, 92 (1996); E. H. Simmons, Phys. Rev. D 55, 1678 (1997).

[4] K. Lane and M. Ramana, Phys. Rev. D 44, 2678 (1991); K. Lane and S. Mrenna, Phys. Rev. D 67, 115011 (2003).

[5] L. Randall and R. Sundrum, Phys. Rev. Lett. 83, 3370 (1999); J. Bijnens et al., Phys. Lett. B 503, 341 (2001).

[6] E. Eichten, I. Hinchliffe, K. Lane, and C. Quigg, Rev. Mod. Phys. 56, 579 (1984); 58, 1065 (1986).

[7] J. L. Hewett and T. G. Rizzo, Phys. Rep. 183, 193 (1989).

[8] R. N. Mohapatra and J.C. Pati, Phys. Rev. D 11, 566 (1975); G. Senjanovic and R. N. Mohapatra, Phys. Rev. D 12, 1502 (1975); R. N. Mohapatra and G. Senjanovic, Phys. Rev. D 23, 165 (1981).

[9] J. Alitti et al. (UA2 Collaboration), Nucl. Phys. B400, 3 (1993).

[10] F. Abe et al. (CDF Collaboration), Phys. Rev. D 55, R5263 (1997).

[11] F. Abe et al. (CDF Collaboration), Phys. Rev. Lett. 74, 3538 (1995).

[12] V. M. Abazov et al. (D0 Collaboration), Phys. Rev. D 69, 111101 (2004).

[13] A. Abulencia et al. (CDF Collaboration), Phys. Rev. Lett. 96, 122001 (2006).

[14] A. Abulencia et al. (CDF Collaboration), Phys. Rev. D 74, 071103 (2006).

[15] A. Abulencia et al. (CDF Collaboration), Phys. Rev. D 75, 092006 (2007).

[16] V. M. Abazov et al. (D0 Collaboration), Phys. Rev. Lett. 101, 062001 (2008).

[17] T. Aaltonen et al. (CDF Collaboration), Phys. Rev. D 78, 052006 (2008).

[18] D. Stump et al., J. High Energy Phys. 10 (2003) 046.

[19] A. Abulencia et al. (CDF Collaboration), J. Phys. G 34, 2457 (2007).

[20] We use a cylindrical coordinate system with the $z$ coordinate along the proton beam direction, the polar angle $\theta$, and the origin at the center of the detector. The rapidity, pseudorapidity, transverse energy, and transverse momentum are defined as $y=\frac{1}{2} \ln \left[\left(E+p_{z}\right) /\left(E-p_{z}\right)\right], \quad \eta=$ - ln $\tan (\theta / 2), E_{T}=E \sin (\theta)$, and $p_{T}=p \sin (\theta)$, respectively.

[21] A. Affolder et al. (CDF Collaboration), Phys. Rev. D 64, 032001 (2001).

[22] The four-vector for each calorimeter tower is computed as a sum of vectors for the electromagnetic and hadronic compartments of the tower; the vector for each compartment is defined by assigning a massless vector with magnitude equal to the deposited energy and with direction from the primary event vertex to the center of each compartment.

[23] G. Blazey et al., arXiv:hep-ex/0005012.

[24] S. D. Ellis, J. Huston, and M. Tonnesmann, eConf C010630, P513 (2001).

[25] The missing $E_{T}$ is defined by $\mathscr{E}_{T}=\left|\vec{E}_{T}\right|, \vec{E}_{T}=-\sum_{i} E_{T}^{i} \hat{\mathbf{n}}_{i}$, where $\hat{\mathbf{n}}_{i}$ is a unit vector perpendicular to the beam axis and pointing at the $i$ th calorimeter tower. The sum $E_{T}$ is defined by $\sum E_{T}=\sum_{i} E_{T}^{i}$. Both sums are over all the calorimeter towers with $E_{T}>100 \mathrm{MeV}$.

[26] A. Bhatti et al., Nucl. Instrum. Methods Phys. Res., Sect. A 566, 375 (2006).

[27] T. Sjostrand et al., Comput. Phys. Commun. 135, 238 (2001).

[28] E. Gerchtein and M. Paulini, eConf C0303241, TUMT005 (2003).

[29] R. Field, Fermilab ME/MC Tuning Workshop (Fermilab, 2002).

[30] A. Affolder et al. (CDF Collaboration), Phys. Rev. D 65, 092002 (2002).

[31] D. Acosta et al. (CDF Collaboration), Phys. Rev. D 71, 112002 (2005).

[32] The final state stable particles in Monte Carlo generators refer to colorless particles having a lifetime greater than $10^{-11} \mathrm{~s}$.

[33] P. Bagnaia et al. (UA2 Collaboration), Phys. Lett. 144B, 283 (1984).

[34] G. Corcella et al., J. High Energy Phys. 01 (2001) 010.

[35] T. Kluge, K. Rabbertz, and M. Wobisch, arXiv:hep-ph/ 0609285.

[36] S. D. Ellis and D. E. Soper, Phys. Rev. D 48, 3160 (1993).

[37] A. D. Martin, R. G. Roberts, W. J. Stirling, and R. S. Thorne, Phys. Lett. B 604, 61 (2004).

[38] H. Davoudiasl, J. L. Hewett, and T. G. Rizzo, Phys. Rev. D 63, 075004 (2001).

[39] R. Hamberg et al., Nucl. Phys. B359, 343 (1991); P. Mathews et al., Nucl. Phys. B713, 333 (2005).

[40] The following parameters are used after private communication with the authors of [4]: $M_{8}^{\prime}=0, M\left(\pi_{22}^{8}\right)=$ $5 M\left(\rho_{T 8}\right) / 6, \quad M\left(\pi_{22}^{1}\right)=M\left(\pi_{22}^{8}\right) / 2, \quad M_{8}=5 M\left(\rho_{T 8}\right) / 6$, and $M(V 8)=\infty$.

[41] L. Demortier, in Proceedings of the Conference on Advanced Statistical Techniques in Particle Physics (Institute for Particle Physics Phenomenology, University of Durham, UK, 2002), p. 18.

[42] V. M. Abazov et al. (D0 Collaboration), Phys. Rev. Lett. 100, 031804 (2008).

[43] T. Aaltonen et al. (CDF Collaboration), Phys. Rev. Lett. 99, 171802 (2007). 